\title{
Identidad de género, ética protestante y atribución de causalidad
}

\author{
Agustín Echebarría Echabe y José F. Valencia Gárate \\ Universidad País Vasco
}

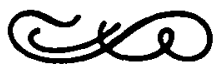

\section{Resumen}

El artículo analiza una doble problemática en tomo a la identidad de género: (a) los determinantes sociales de la identidad de género y (b) los sesgos preceptivos-cognitivos en la evaluación y explicación de acciones desarrolladas por un actor segun su pertenencia sexual.

Se concluye que la génesis de las diferencias en las identidades sociales de bombres y mujeres esta ligada a la distribución social del trabajo en función del sexo. Igualmente se confirma la existencia de sesgos perceptivo-cognitivos en la evaluación de acciones sociales.

Palabras clave: Identidad social de género.

\section{Gender identity, protestant work ethic and the attribution of causality}

\section{Abstract}

This paper analyzes two questions about gender identity: (a) the social origins of gender identity and (b) the cognitive and perceptive bases in judging an action depending on the tanget sex membership.

We conclude that the social distribution of jobs according to sex membership is the cause of differences in gender identity between males and females. Moreover, the cognitive and perceptive bases are confirmed.

Key words: Gender social identity.

Dirección de los autores: Dpto. Psicología Social y Metodología. Facultad Psicología. Avenida de Tolosa, 70. 20009 San Sebastián. 
Identidad de género y valores: ética protestante

La Identidad Social de Género representa un objeto de estudio que posibilita al psicólogo social plantearse un viejo problema dentro de su disciplina: la influencia que ejerce el contexto ideológico y la estructura social en la construcción de la identidad social, por un lado, y en los patrones cognitivos, de otro. En otras palabras, permite abordar el tema de la integración de diversos órdenes de análisis (Doise, 1982).

La presente exposición no trata de ser un artículo estándard en el que se presenta una investigación concreta, sino más bien una reflexión sobre las diferencias entre sexos y la razón histórica de dichas diferencias, así como la complejidad del tema ilustrándose la exposición con resultados de diferentes estudios realizados por nuestro equipo.

La identidad social de género está asociada a un sistema de creencias sociales sobre el género que incluye creencias sobre la masculinidad y la feminidad; opiniones sobre las cualidades, creencias sobre el tipo de actividad adecuada para cada sexo, así como la distribución de ocupaciones según la pertenencia sexual (Deaux y Kite, 1987; Hartman et al., 1988; Flament, 1989; Echebarría, 1992).

Dentro de este sitema de creencias de género, la identidad femenina ha sido caracterizada como dependiente, débil, afectiva, comprensiva, complaciente, centrada en los demás; mientras que la identidad masculina ha sido definida como ambiciosa, fuerte, competitiva, agresiva, independiente, autosuficiente, etc. (Lorenzi-Cioldi, 1988; Deaux y Kite, 1987; Echebarría, 1992; Echebarría y Valencia, 1992).

Intentando hallar los orígenes de esta diferenciación, algunos autores señalan que la misma proviene de la división entre el ámbito de la actividad pública y privada que tiene lugar a raíz de la revolución industrial (Thoits, 1989). En esta distribución de los roles sociales, la mujer va a quedar encargada del ámbito de lo privado (el hogar y la familia), mientras que el hombre se va a ocupar del ámbito de lo público (trabajo) (Parsons y Bales, 1955). En otras palabras, la mujer va a asumir funciones de carácter emocional (apoyo a los suyos) y centradas en el propio grupo. Por lo contrario, el hombre va a desarrollar funciones instrumentales centradas fuera del grupo. Con el transcurso del tiempo, las actividades características de cada sexo según su ámbito de actuación (privado versus público) serán vistas como reflejo de características personales de los ocupantes de dichos roles. La distribución social de los roles sociales sería, en este sentido, suficiente para explicar la formación de los estereotipos sexuales, permitiendo mantener los mismos (Eagly y Steffen, 1984).

Hoffman y Hurst (1990) plantean la bipótesis de la racionalización, según la cual dichos estereotipos sexuales cumplirían funciones de racionalización, justificación o explicación de la división del trabajo en función del sexo, asumiendo que existen diferencias entre ellos que hacen a un sexo más apto que al otro para ciertas ocupaciones, y viceversa. Además, el estereotipo femenino, menos valorado socialmente, permite, en la medida en que es interiorizado por la mujer, mantener a ésta en una situación de status inferior (Breakwell, 1990).

En un estudio realizado por nuestro equipo (Echebarría, 1993) en la que tomaron parte 197 sujetos ( 90 hombres y 107 mujeres; media de edad $=47$ años) se encontró una fuerte relación entre el tipo de tarea desempeñada y la identidad social de género de sus ocupantes (Chi-Cuadrado $=31.86$; G. L. $=3$; $\mathrm{p}=0.0000$ ). La distribución de identidades según las ocupaciones (categoriza- 
das como tradicionalmente masculinas o femeninas) fue la que se recoge en la Tabla I.

TABLA I

Identidades sociales de género.

\begin{tabular}{lcccc}
\hline & Indif. & Androg. & Masculina & Femenina \\
FEMENINA & 24 & 24 & 2 & 28 \\
TIPO DE & $(30,8 \%)$ & $(30,8 \%)$ & $(2,6 \%)$ & $(35,9 \%)$ \\
\cline { 2 - 5 } OCUPACION & & & & \\
MASCULINA & 20 & 17 & 27 & 11 \\
& $(25,6 \%)$ & $(24,4 \%)$ & $(36,0 \%)$ & $(14,0 \%)$ \\
\hline
\end{tabular}

Nota: Los estudiantes (25), jubilados (11) y parados (8) fueron excluidos.

Resumiendo, la distribución desigual del trabajo entre hombres y mujeres sería la causa histórica de la génesis de los estereotipos actualmente existentes sobre los sexos. A través de procesos de autoestereotipaje dichos estereotipos serían interiorizados por los sujetos como propios de su identidad social. El proceso de auto-estereotipaje hace referencia a «los procesos cognitivos que conducen a un sujeto a percibirse a sí mismo como miembro de un grupo, y a comportarse de acuerdo con la identidad social resultante» (Lorenzi-Cioldi, 1991, p. 403).

Pero además de lo anterior, la distribución de los roles sociales entre el hombre y la mujer va a tener incidencia en términos de socialización de valores (Echebarría, 1992). Ya Weber (1980) señaló que el desarrollo del capitalismo incipiente va parejo al desarrollo de una concepción ideológico-religiosa que posibilita y legitima al mismo: la Etica Protestante y el Calvinismo. El ascetismo protestante inicial subraya (Feather, 1984, pag. 1.132) que «las virtudes del trabajo duro, la autodisciplina, la negación del placer por el placer» son los medios para desarrollarse como persona. Si bien la búsqueda de la riqueza en sí misma es negativa, el logro de ella a través del trabajo es signo de bendición de Dios. Este marco ideológico religioso va a impulsar un cambio en la concepción de la persona. Va a estimular una visión «egocéntrica o individualista» de la persona que será percibida como autónoma, agente de transformación del entorno, rodeada de una barrera que la separa de la sociedad, con un mundo interior propio y auténtico reflejo de su yo (Schweder y Bourne, 1984a, 1984b; Echebarría, 1991). Este marco va a potenciar una concepción de autoresponsabilidad individual sobre los propios actos, creencias internas de control personal sobre el medio y va a estimular un sistema de valores que enfatiza el éxito económico y social (Farr, 1987; Andersen, 1987; Logan, 1987). El sujeto socializado en esta Etica Protestante va a aparecer como sujeto con fuertes necesidades de logro personal, competitivo, ambicioso, y con estilos atribucionales internos (Feather, 1985; Mirels y Garrett, 1971; Furnham y Quilley, 1989; Echebarría, 1992). Esta concepción de autoresponsabilidad va a ser transmitida a través de las prácticas evaluativas por los agentes de socialización (Le Poultier, 1989; Beauvois y Le Poultier, 1986; Joule y Beauvois, 1986).

Ahora bien, la socialización en este sistema de valores derivados de la Etica Protestante no va a ser homogénea en todos los estratos sociales. Por ejemplo, esta percepción interna sobre el control de los acontecimientos y sucesos que 


\section{8}

nos rodean será más acusada entre los sujetos de clases sociales más favorecidas (Dubois, 1986). Además, este sistema de valores es especialmente funcional en el ámbito de lo público (logro en el trabajo). Esto nos lleva a pensar que serán los hombres sobre los que la socialización ejerza mayor presión para la interiorización de dichos valores. Confirmando este hecho nos encontramos por una parte que las características que definen al sujeto adherido a los valores de la Etica Protestante (ambicioso, competitivo, agresivo, independiente, con fuerte motivación de logro) (Feather, 1985) se corresponden al estereotipo clásico de lo masculino.

En el estudio anteriormente citado (Echebarría, 1993) se encontraron diferencias en valores entre los sujetos según su identidad de género. Así, los esquematizados masculinos fueron los que más fuertemente se adherían a los valores que enfatizaban la importancia del logro en el ámbito ocupacional $(\mathrm{F}(3,193)=2.66, \mathrm{p}=0.04)$. También en dicho estudio se encontró una clara asociación entre la identidad social de género masculina, la ocupación en tareas tradicionalmente caracterizadas como masculinas, la pertenencia sexual al grupo varón, creencias más internas de control y estilos atribucionales de carácter egodefensivo (asumir responsabilidades por los éxitos y rechazar las responsabilidades por los fracasos). Estos resultados pueden resumirse gráficamente como sigue:

\section{GRAFICO 1}

Análisis de correlación canónica.;

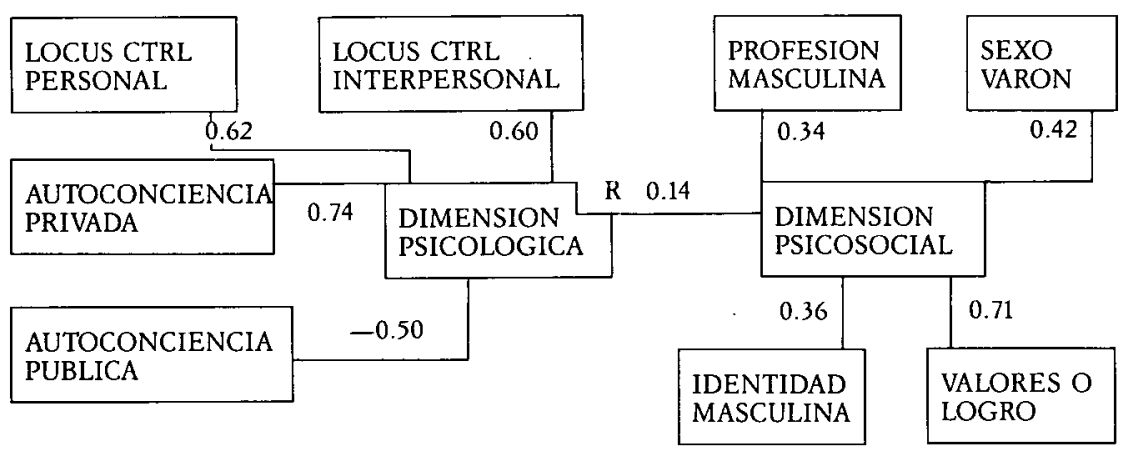

Correlaciones entre las dimensiones del análisis de correlación canónica y contextos de atribución.

\begin{tabular}{|c|c|c|}
\hline CONTEXTOS DE ATRIBUCION & $\begin{array}{l}\text { DIMENSIÓN } \\
\text { PSICOLOGICA }\end{array}$ & $\begin{array}{l}\text { DIMENSION } \\
\text { PSICOSOCIAL }\end{array}$ \\
\hline $\begin{array}{l}\text { ATRIB. EXTERNA EN } \\
\text { CONTEXTO POSITIVO }\end{array}$ & $-0.37^{*}$ & $-0.38^{*}$ \\
\hline $\begin{array}{l}\text { ATRIB. EXTERNA EN } \\
\text { CONTEXTO NEGATIVO }\end{array}$ & $0.13^{\star}$ & $0.95^{*}$ \\
\hline $\begin{array}{l}\text { ATRIB. INTERNA EN } \\
\text { CONTEXTO POSITIVO }\end{array}$ & $0.64^{\star}$ & 0.06 \\
\hline $\begin{array}{l}\text { ATRIB. INTERNA EN } \\
\text { CONTEXTO NEGATIVO }\end{array}$ & $-0.77^{\star}$ & $-0.12^{*}$ \\
\hline
\end{tabular}

\footnotetext{
* Significación de R 0.05 .
} 
Como se puede observar, y dejando al margen la autoconciencia que no nos ocupa en la presente exposición, podemos ver que el análisis de correlación canónica extrajo dos dimensiones. La primera, que bautizamos «psicológica», porque agrupa a los elementos más cognitivos como el locus de control (y la autoconciencia), y la segunda, denominada «psicosocial», porque agrupa elementos psicosociales como la distribución ocupacional o la pertenencia sexual, la identidad social de género o los valores. El perfil que obtenemos ilustra las afirmaciones anteriores: la correspondencia entre el desempeño de ocupaciones «masculinas" con el desarrollo de una identidad de género masculina, y la interiorización de valores de logro socioocupacionales.

Ahora bien, si todo lo expuesto anteriormente fuese correcto, podría inferirse lógicamente que la distribución de las identidades sociales de género entre hombres y mujeres debería irse transformando a medida que la mujer va accediendo también al ámbito de lo público. La reestructuración de la distribución del trabajo debería conducir, especialmente en la mujer, pero también en el varón, a cambios profundos de identidad. Podría pensarse que si comparásemos la distribución de identidades de género en muestras de mayor edad con otras más jóvenes debería ofrecernos una panorámica bien distinta. Al menos en el estado español, los cambios producidos en cuanto al acceso de la mujer a ámbitos anteriormente restringidos a los varones desde los años 50 y 60 a la actualidad han sido muy importantes. Esta afirmación se ve corroborada si comparamos los resultados del estudio anterior (Echebarría, 1993), en el que tomaron parte 197 personas, con una media de edad de 47 años, con el estudio llevado a cabo por Echebarría y Valencia (1992), en el que la muestra se compuso de 432 jóvenes cuya media de edad fue de 14 años. Las distribuciones de identidades sociales de género según la pertenencia sexual de los sujetos de cada muestra se presenta en la Tabla II.

TABLA II

(a) MUESTRA ADULTA ( $\mathrm{N}=197$, MEDIA EDAD = 47 AÑOS).

\begin{tabular}{ccccc}
\hline \multicolumn{5}{c}{ IDENTIDADES DE GENERO } \\
& INDIFERENC. & ANDROGIN. & MASCULINA & FEMENINA \\
\hline HOMBRE & $\begin{array}{c}22 \\
(24,4 \%)\end{array}$ & $\begin{array}{c}25 \\
(27,8 \%)\end{array}$ & $\begin{array}{c}33 \\
(36,7 \%)\end{array}$ & $\begin{array}{c}10 \\
(11,1 \%)\end{array}$ \\
\hline \multirow{2}{*}{ MUJER } & 32 & 34 & 7 & 34 \\
& $(29,9 \%)$ & $(31,8 \%)$ & $(6,5 \%)$ & $(31,8 \%)$ \\
\hline
\end{tabular}

CHI-CUADRADO $=31.98, \mathrm{P}=0.0001$

(b) MUESTRA JOVEN ( $\mathrm{N}=432$, MEDIA EDAD $=14$ AÑOS).

IDENTIDADES DE GENERO

INDIFERENC. ANDROGIN. MASCULINA FEMENINA

\begin{tabular}{ccccc}
\hline HOMBRE & $(43,6 \%)$ & $(8,3 \%)$ & $(19,6 \%)$ & $(28,4 \%)$ \\
\hline MUJER & $(6,9 \%)$ & $(46,3 \%)$ & $(28,2 \%)$ & $(18,6 \%)$ \\
\hline
\end{tabular}

CHI-CUADRADO $=110.78, \mathrm{P}=0.0001$. 
El carácter comparativo de ambas muestras y el tipo de estudios imposibilita realizar con rigor afirmaciones sobre las causas que han conducido a este cambio dramático. Sin embargo, creemos que la profunda transformación social y el tipo de expectativas ocupacionales que poseían y poseen los sujetos de ambas muestras puede desempeñar un papel importante en el mismo. Como vemos, mientras que los varones parecen inclinarse hacia una progresiva indiferenciación en términos de identidad de género, las mujeres, sin abandonar las características femeninas tienden a incorporar atributos clásicamente asociados al estereotipo masculino, manifestando una tendendia clara hacia la androginia. Podría pensarse que este dato va en la línea de lo afirmado por Amâncio (1989) cuando señala que las mujeres, en tanto que miembros de grupos socialmente dominados, son conscientes de la irrelevancia de las características femeninas para el logro de una diferenciación intergrupal positiva como una distintividad individual. Por ello, su estrategia se basaría en «competir» con el exogrupo varón sobre las dimensiones de comparación propias de dicho exogrupo.

En los resultados de los estudios anteriores parece que la distribución de identidades sociales a ambos sexos se va modificando en las nuevas generaciones, pensamos que en gran parte debido a las transformaciones sociales en la distribución social del trabajo entre hombres y mujeres. ¿Significa esto que los estereotipos anteriores han dejado de ejercer su influencia en la interpretación que hacemos de los demás en contextos cotidianos?

\section{ESTEREOTIPOS SEXUALES, ATRIBUCION Y PERCEPCION SOCIAL}

Como afirman Duveen y Lloyd, las etiquetas de hombre y mujer son categorías sociales relevantes porque (a) ejercen una influencia constante en los encuentros sociales, (b) sirven para categorizar de forma exhaustiva a todo ser humano y (c) la pertenencia sexual es saliente y altamente visible. Las representaciones de género nos proveen de un marco explicativo para interpretar las acciones de los demás. Futoran y Wyer (1986) señalaron el impacto del etiquetaje sexual sobre juicios de adecuación a una ocupación. El etiquetaje sexual ejercía mayor impacto sobre el juicio que la información sobre atributos del candidato/a. Hartman, Griffeth, Miller y Kinicki (1988) encontraron que la ejecución de un hombre en una tarea masculina o de una mujer en una femenina eran explicadas por elementos estables como la habilidad. Sin embargo, cuando dicha correspondencia entre actor y tarea no se produce, predominaban las explicaciones inestables, como la suerte. En una línea similar, Löchel (1983) afirma que los éxitos en los varones son atribuidos a las habilidades mientras que en las mujeres se atribuyen a la suerte. Por lo contrario, en situaciones de fracaso, la falta de habilidad como explicación predomina cuando el actor es una mujer.

Murphy-Berman y Sharma (1988) encuentran un estilo atribucional autoderogatorio en las mujeres. Estas manifestarían atribuciones más externas que los hombres para los éxitos, pero no para los fracasos. Para no extendernos en la revisión, señalar que las tareas masculinas suelen valorarse más que las femeninas (Hartman et al., 1988).

A continuación presentaremos los resultados de un estudio actualmente en fase de análisis, por lo que dichos resultados deben tomarse como provisionales, que ilustra el impacto de las representaciones de género en los juicios causales y la percepción de la ejecución en una tarea según quien sea su actor. 
La muestra se compuso de 117 sujetos cuyas edades oscilaban entre los 14 y los 45 años. A estos sujetos se les presentó una historia ficticia que describía a un hombre versus una mujer, realizando una tarea (masculina versus femenina), con un resultado exitoso versus fracaso. Así se obtuvo un diseño factorial $2 \times 2 \times 2$. Previamente a la lectura de la historia los sujetos respondieron al BSRI para evaluar su identidad de género.

Tras la lectura de la historia se pidió a los sujetos en formato abierto que respondiesen a 2 preguntas: «cuál cree usted que es la causa de... (resultado exitoso o fracaso)?», y "¿cómo describiría usted a... ?» (actor de la historia).

Se elaboró un diccionario con las categorías mencionadas para responder a cada pregunta. Posteriormente se seleccionaron aquellas categorías mencionadas al menos por el $10 \%$ de los sujetos experimentales.

Previamente a la realización de los análisis de varianza correspondientes, se realizaron dos análisis de Princals, uno para las categorías de respuesta para la explicación causal, y el otro para las categorías mencionadas para decribir al actor. El análisis de Princals realiza un análisis de componentes principales mediante el método de mínimos cuadrados. Analiza un número de categorías para extraer dimensiones subyacentes. A diferencia del análisis factorial tradicional, no requiere que las variables estén medidas en intervalos (de hecho ha sido diseñado para datos ordinales y nominales), y tampoco asume que las relaciones entre las variables sean lineales. Finalmente, permite salvar las dimensiones encontradas, permitiendo someter a las mismas a análisis de varianza posteriores. Los resultados de dicho análisis para las categorías asociadas a ambas preguntas se recogen en la Tabla III.

Como se puede observar, la primera dimensión de atribución opone las explicaciones en términos de competencia, responsabilidad y asertividad a desorganización y causas externas. La segunda dimensión opone competencia y responsabilidad a dar una buena impresión pública y agrado por la actividad realizada. La tercera dimensión opone causas externas a desorganización, incompetencia y método. Finalmente, la cuarta dimensión opone el método a la incompetencia.

\author{
TABLA III
}

Análisis Princals.

(a) EXPLICACIONES CAUSALES

\begin{tabular}{|c|c|c|c|c|}
\hline \multirow[t]{2}{*}{ VARIABLES } & \multicolumn{2}{|c|}{ DIMENSIONES } & \multirow[b]{2}{*}{3} & \multirow[b]{2}{*}{4} \\
\hline & 1 & 2 & & \\
\hline Competencia & 0.586 & -0.393 & & \\
\hline Responsabilidad & 0.655 & -0.308 & & \\
\hline Asertividad & 0.513 & & & \\
\hline Desorganización & -0.621 & & -0.527 & \\
\hline Causas externas & -0.490 & & 0.339 & \\
\hline Dar buena impresión & & 0.817 & & \\
\hline Gusta lo que hace & & 0.541 & & \\
\hline Incompetencia & & & -0.508 & -0.657 \\
\hline Método & & & -0.630 & 0.682 \\
\hline
\end{tabular}


(b) PERCEPCION DEL ACTOR

VARIABLES
DIMENSIONES

2
Irresponsable

Descuidado

Inmaduro

Competente

Trabajador

Buen aspecto

Asertivo

Maduro

Autoritario

Metódico
0.541

0.595

0.390

$-0.474$

$-0.630$

$-0.328$

$-0.472$

$-0.424$
0.360

0.727

0.456

En cuanto a la descripción del actor, la primera dimensión opone irresponsable, descuidado e inmaduro a competente, trabajador, asertivo, maduro y buena imagen física. La segunda dimensión opone autoritario, inmaduro pero metódico a asertivo.

Posteriormente se realizaron seis análisis de varianza (uno para cada una de las dimensiones anteriores) para analizar el impacto del actor (mujer versus hombre), el tipo de tarea (masculina versus femenina) y el resultado de la misma (éxito versus fracaso) en los juicios causales y la percepción del actor. En la Tabla IV se recogen los efectos significativos resultantes de tales análisis.

\section{TABLA IV}

(a) Comenzando con el efecto principal de la pertenencia sexual del actor, podemos observar lo siguiente:

- La ejecución del actor «hombre» es atribuida en mayor medida a la competencia, la responsabilidad y la asertividad mientras que la ejecución del actor «mujer» se atribuye a la desorganización y a causas externas (primera dimensión de atribución) $(\mathrm{Xh}=0.44$ vs. $\mathrm{Xm}=-0.46$ ).

- Centrándonos en la segunda dimensión de atribución, la ejecución de la mujeres se atribuye al deseo de dar una buena impresión pública y el agrado por la actividad realizada, mientras que en el caso del hombre la atribución es de responsabilidad y competencia $(\mathrm{Xh}=-0.11$ vs. $\mathrm{Xm}=0.17$ ).

- Por lo que respecta a la tercera dimensión de atribución, la ejecución de la mujer se atribuye a la desorganización, la incompetencia y el método, mientras que en el hombre se atribuye a causas externas.

- Finalmente, centrándonos en la cuarta dimensión de atribución, la ejecución del varón se atribuyó al método, mientras que la de la mujer,a la incompetencia.

- Por lo que respecta a la descripción del actor (primera dimensión), el hombre fue descrito como competente, trabajador, maduro, asertivo y de buena apariencia $(X=-0.15)$; mientras que la mujer fue descrita como irresponsable, inmadura y descuidada $(X=0.15)$.

\section{(b) Efecto principal del tipo de tarea (masculina versus femenina):}

- Comenzando con la primera dimensión de atribución, la ejecución en una tarea masculina se explicó en términos de competencia, responsabilidad y asertividad ( $X=0.19$ ), 
mientras que la ejecución en una tarea femenina se explicó en términos de causas externas y desorganización $(X=-0.15)$.

- Por lo que respecta a la segunda dimensión de atribución, la tarea femenina se explicó por el deseo de dar una imagen pública positiva y el agrado por la tarea realizada $(X=0.41)$; mientras que la ejecución en la tarea masculina se atribuyó a la competencia y la responsabilidad $(\mathrm{X}=-0.49)$.

- Tercera dimensión de atribución: la tarea femenina se explicó por causas externas $(\mathrm{X}=0.34)$, mientras que la masculina,por la desorganización, la incompetencia y el método $(\mathrm{X}=-0.36)$.

- Finalmente, por lo que respecta a la percepción (segunda dimensión) el actor en una tarea femenina fue descrito como asertivo $(X=0.43)$, mientras en la tarea masculina lo fue en términos de autoritarismo, metódico e inmaduro $(\mathrm{X}=-0.30)$.

(c) Interacción del sexo del actor y el tipo de tarea:

TABLA $\mathrm{V}$

\begin{tabular}{cccc}
\hline & & \multicolumn{2}{c}{ TIPO DE TAREA } \\
& & FEMENINA & MASCULINA \\
\hline \multirow{3}{*}{ ACTOR } & HOMBRE & -0.82 & 1.02 \\
& MUJER & 0.43 & -0.71 \\
\hline
\end{tabular}

Atribución: primera dimensión.

Observando las medias en la primera dimensión de atribución de la Tabla $V$ observamos que la ejecución de un actor varón en una tarea masculina y en menor medida la de una mujer en una tarea femenina se explicaron por la responsabilidad, la competencia y la asertividad. Por lo contrario, las ejecuciones en las situaciones de no correspondencia entre sexo del actor y tipo de tarea se explicaron por la desorganización y causas externas.

(d) Interacción entre tipo de tarea y sexo del actor: segunda dimensión de atribución:

TABLA VI

\begin{tabular}{cccc}
\hline & & \multicolumn{2}{c}{ TIPO DE TAREA } \\
& & FEMENINA & MASCULINA \\
\hline \multirow{3}{*}{ ACTOR } & HOMBRE & -0.28 & -0.66 \\
& MUJER & 0.99 & -0.31 \\
\hline
\end{tabular}

En la Tabla VI podemos ver que la ejecución de un varón en una tarea masculina y en menor medida en una femenina se explicó por la responsabilidad y la competencia, mientras que la ejecución de una mujer en una tarea femenina se explicó por el deseo de dar una buena imagen y el agrado por la actividad. 


\section{4}

(e) Interacción del tipo de tarea y el sexo del actor sobre la percepción (primera dimensión) TABLA VII

\begin{tabular}{cccc}
\hline & & \multicolumn{2}{c}{ TIPO DE TAREA } \\
& & FEMENINA & MASCULINA \\
\hline \multirow{3}{*}{ ACTOR } & HOMBRE & 0.31 & -0.64 \\
& MUJER & -0.32 & 0.78 \\
\hline
\end{tabular}

En la Tabla VII podemos observar que el actor hombre realizando una tarea masculina y la mujer en una tarea femenina fueron descritos como competentes, trabajadores, asertivos, maduros y de buena imagen, mientras que cuando la correspondencia entre sexo del actor y tipo de tarea no se producía, la descripción era en términos de irresponsabilidad, descuidados e inmaduros.

(f) Interacción del resultado (éxito versus fracaso) y el sexo del actor sobre la atribución (cuarta dimensión):

\section{TABLA VIII}

\begin{tabular}{cccc}
\hline & & \multicolumn{2}{c}{ SEXO DEL ACTOR } \\
& & MUJER & HOMBRE \\
\hline \multirow{2}{*}{ RESULTADO } & FRACASO & -0.32 & -0.49 \\
& EXITO & 0.50 & -0.41 \\
\hline
\end{tabular}

Como puede observarse, el resultado del varón fue descrito en términos de competencia (incompetencia) independientemente del resultado, mientras que el éxito de la mujer se atribuyó al método, mientras el fracaso a la incompetencia.

Como conclusión global podemos observar que:

(a) La ejecución del varón es explicada en términos de competencia, responsabilidad y trabajo en mayor medida que la ejecución de la mujer.

(b) Lo mismo se puede aplicar para el caso de las tareas masculinas frente a las femeninas.

(c) Finalmente, en situaciones en las que existe una correspondencia entre el sexo del actor y el tipo de trabajo que desempeñan, éstos son descritos como maduros, trabajadores y competentes, lo que no sucede cuando dicha correspondencia no se da.

Para simplificar la exposición del estudio, intentando encontrar patrones atribucionales y de percepción, se realizó en análisis de Princals incluyendo además de las categorías utilizadas en la explicación de la viñeta y la descripción del actor el sexo del actor, la identidad de género del sujeto experimental, el tipo de tarea y el resultado de la misma. Los resultados se presentan en la tabla siguiente. 
TABLA IX

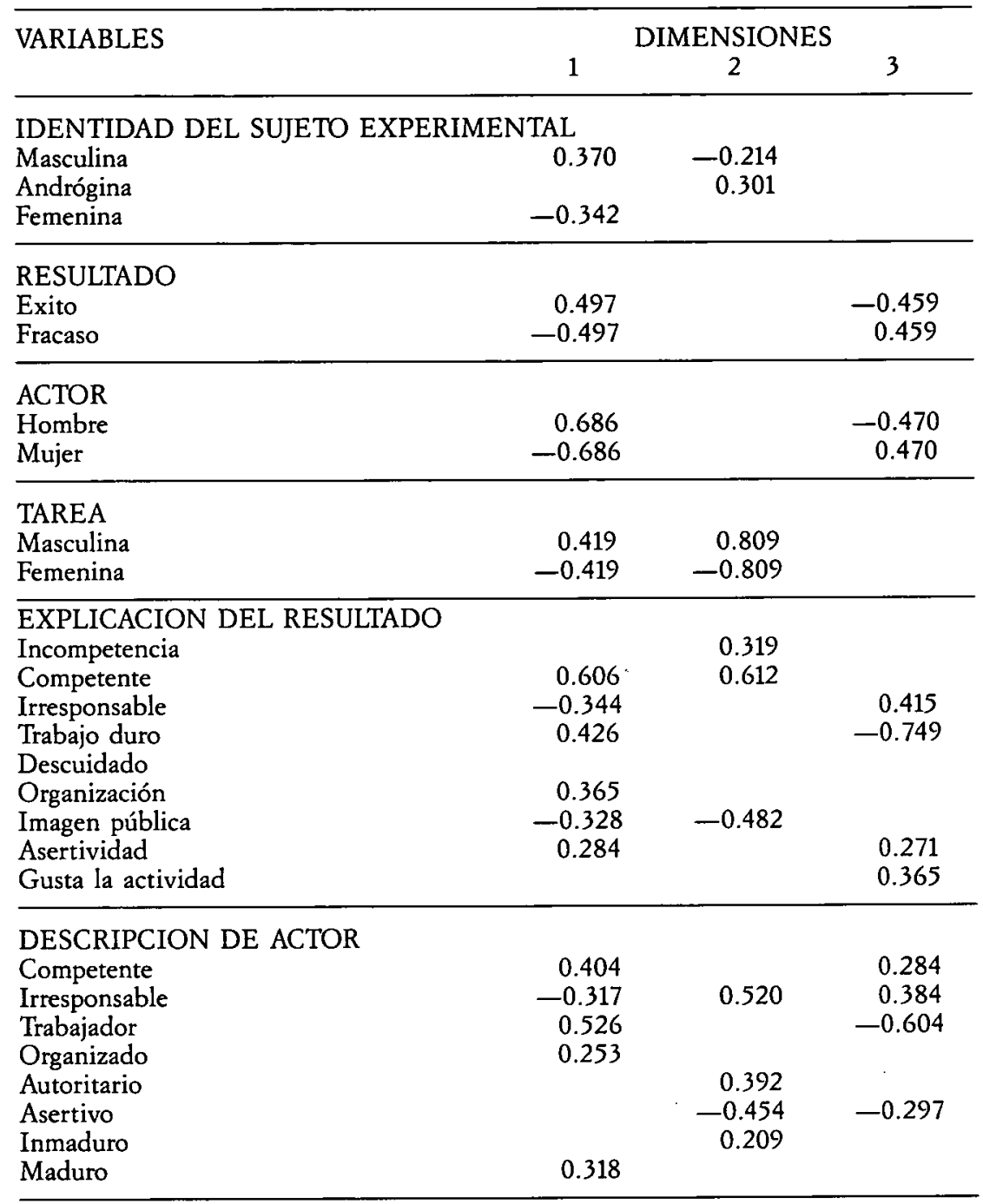

Como se puede observar en la tabla anterior, la primera dimensión opone: Observador con identidad masculina, leyendo una viñeta en la que un hombre ejecuta exitosamente una tarea masculina, siendo descrito como competente, trabajador, maduro y organizado, y atribuyendo la ejecución al trabajo, organización y competencia por un lado, a: observador con identidad femenina que lee una viñeta en la que una mujer ejecuta sin éxito una tarea femenina, que es descrita como irresponsable, y se explica la tarea por el deseo de dar una buena imagen, y la irresponsabilidad.

La segunda dimensión opone: (a) observador andrógino, leyendo una viñeta en la que se describe una tarea masculina, explicada en términos de incompetencia y competencia, y describiendo al actor en términos de autoritario, inma- 


\section{6}

duro y organizado; a (b) un observador masculino, leyendo una viñeta que describe una tarea femenina, explicado en términos de deseo de dar una buena impresión pública, y describiendo al actor en términos de asertividad.

La tercera dimensión opone: (a) actor mujer fracasando, siendo descrita como competente pero irresponsable, y explicándose la ejecución en términos de irresponsabilidad, agrado por la actividad y asertividad; a (b) hombre exitoso, descrito como trabajador y asertivo, explicándose el éxito por el trabajo.

\section{CONCLUSIONES}

De lo anteriormente expuesto pueden extraerse una serie de conclusiones.

(a) En primer lugar, resaltar la importancia que desempeña la organización social (distribución desigual del trabajo) en la génesis y mantenimiento de las creencias sociales sobre la masculinidad y la feminidad. Esta determinación social es en ocasiones olvidada. Ciertas tendencias que han dirigido el estudio de la masculinidad y la feminidad únicamente al terreno de la detección de diferencias interindividuales en términos de identidades, estructuras cognitivas y diferencias en la integración de la información, corre el riesgo, al no contemplar la dimensión social del problema, de «reificar» las diferencias entre sexos, substituyendo desde un prisma cognitivo la antigua legitimación de las desigualdades en términos de determinismo biológico. En otras palabras, el determinismo cognitivo corre el riesgo de cumplir las funciones que anteriormente cumplió el biológico.

(b) En el estudio experimental se manifiesta el mantenimiento de ciertas formas estereotípicas de evaluar e interpretar la situación cuando la categorización en términos de pertenencia sexual se hace manifiesta.

\section{Referencias}

Andersen, S. M. (1987). The role of cultural assumptions in Self concept development. K. Yardley and T. Honess (Eds.). Self and Identity: Psychosocial perspectives, Nueva York: John Wiley \& Sons.

Amancio, L. (1989). Social differentiation between «dominant» and «dominated» groups: Towards an integration of Social Stereotypes and social identity. European Joumal of Social Psychology, vol. 19, n. $1: 1-10$.

Barrios, P.; Echebarrí. A; Martínez, B., y Txurimendi, F. (1986). Instrumentos para medir síntomas ansiosos y depresivos. D. Páez et al. (Eds.). Salud mental y factores psicosociales, Madrid: Fundamentos.

Beauvois, J. L., y Dubois, N. (1988). The norm of internality in the explanation of psychological events. European Joumal of Social Psychology, 18 (4), 299-316.

Beauvois, J. L., y Le Poultier, F. (1986). Norme d'internalité et pouvoir social en psychologie quotidienne. Psychologie Francaise, 31-2, 100-108.

Breakwell, G. M. (1990). Social beliefs about gender differences. C. Fraser \& G. Gaskell (Eds.). The social psychological study of widespread beliefs, Nueva York, Oxford University Press.

Deaux, K., y Kite, M. E. (1987). Thinking about gender. B. B. Hess and M. Marx Ferree (Eds.). Analyzing gender. A bandbook of social science research, Beverly Hills: SAGE.

Dorse, W. (1982). L'Explication en Psychologie Sociale, París, Presses Universitaires de France.

Dubors, N. (1986). Aspects normatifs versus cognitifs de l'evolution de l'enfant vers la norme d'internalité. Psychologie Française, n.0 31-2: 109-114.

Duveen, G., y LLOYd, B. (1986). The significance of social identities. British Joumal of Social Psychology, vol. 25: 219-230.

Eagly, A. H., y StefFen, V. S. (1984). Gender stereotypes stem from the distribution of women and men into social roles. Joumal of Personality and Social psychology, 46 (4), 735-754.

Echerarrí, A. (1986). Factores psicosociales de riesgo en las candiopatías isquémicas coronarias: Aspectos teóricos y metodológicos, Bilbao: Edit. Central de la Universidad del País Vasco.

Echebarria, A. (1991). El self. A. Echebarría (Ed.), Psicología social sociocognitiva, Bilbao, Desclée de Brouwer. 
ECHEBARRiA, A. (1993). Gender social identity, values and cognitive styles. Revue Intemationale de Psychologie Sociale, $T 5, n^{\circ} 2$.

Echebarría, A., y Valencia, J. F. (1992). Identidad social de género, evaluaciones intercategoriales y percepción social. Revista de Psicología Social (en prensa).

Echebarria, A.; Martinez, B.; Páez, D., y Valencia.J. F. (1987). Autoconciencia, conducta y emoción. Boletín de Psicología, 15, 83-117.

FARR, R. (1987). Individualism as a collective representation. Colloque sur «ideologies et representations sociales», Université Paris-X, Nanterre.

FeAther, N. T. (1985). Attitudes, values and attribution: Explanations of unemployment. Joumal of Personality and Social Psychology, vol. 48: 876-889.

FeATHER, N. T. (1984). Masculinity, feminity, psychological androgyny, and the structure of values.Joumal of Personality and Social Psychology, 47 (3), 604-620.

Fenigstein, A. (1979). Self-consciousness, self-attention and social interaction. Joumal of Personality and Social Psychology, 37, 75-86.

Fenigstein, A.; Scheier, M. F., y Buss, A. H. (1975). Public and private self-consciousness: Assessment and Theory. Joumal of Consulting and Clinical Psychology, 43 (4), 522.527.

Flament, J. C. (1989). Structure et dynamique des representations sociales. D. Jodelet (Ed.). Les representations sociales, París: Presses Universitaires de France.

Furnham, A., y Quiley, R. (1989). The protestant work ethic and the prisioner's dilemma game. Brithish Joumal of Social Psychology, vol. 28: 79-87.

Furnham, A. (1984). The Protestant Work Ethic. European Joumal of Social Psychology, 14, 87-104.

FutORAN, G., y WYYR, S. (1986). The effects of traits and gender stereotypes on occupational suitability judgments and the recall of judgment-relevant information. Joumal of Experimental Social Psychology, 22, 475-503.

Hartman. S. J., et al. (1988). The impact of occupation, performance and sex on sex role stereotyping. Joumal of Social Psychology, 128 (4), 451-463. .

Hoffman, C., y Hurst, N. (1990). Gender Stereotypes. Perception or razionalization? Joumal of personality and Social Psychology, vol. 58, n. ${ }^{\circ}$ 2: 197-208.

IcKes, W., y LAYDEN, M. A. (1976). Attributional styles. H. G. Harvey; W. Ickes and M. A. Weary (Eds.). New Directions in attribution research, Hillsdale: Lawrence Erlbaum Ass. Pub.

JAHODA, G. (1988). J'Accuse. M. Harris (Ed.). The cross cultural challenge to social psychology, Beverly-Hills: SAGE.

Joule, R., y Beauvors, J. L. (1986). Prediction et explanations d'un comportment de soumission. Psychologie Française, n. $31-2$ : 149-155.

Kenny, D. (1989). Correlation and causality, Nueva York: Wiley \& Sons.

LE Poutrier, F. (1989). Acquisition de la nome d'internalité et activité evaluative. J. L. Beauvois; R. Joule and J. M. Monteil (Eds.). Perspectives cognitives et conduites sociales, 2, Fribourg: Del Val.

LöCHEL, E. (1983). Sex differences in achievement motivation. J. C. Jaspars; F. D. Hincham and M. Hewstone (Eds.). Attribution theory and research: Conceptual, developmental and social dimensions, Londres: Academic Press.

Logan, R. D. (1987). Historical change in prevailing sense of self. K. Yardley \& T. Honess (Eds). Self and identity: Psychological perspectives, Nueva York, John Wiley \& Sons.

Lorenzi.Cioldi, F. (1988). Individus dominants et groupes dominés. Images masculines et femenines, Grenoble, Presses Universitaires de Grenoble.

LORENZI.CIOLDI, F. (1991). Self-stereotyping and self-enhacement in gender groups. European Jour. nal of Social Psychology, 21: 403-417.

Miller, S. M., y Seligman, E. P. (1984). Modelo reformulado del desamparo y la depresión. W. S. Neufeld (Ed.). Psicopatologia y stress, Barcelona: Toray.

Mirels, H., y GarReTt.J. (1971). The protestant ethic as a personality variable. Joumal of Consulting and Clinical psychology, vol. 36: 40-44.

Murphy.Berman, W., y Sharma, R. (1988). Testing the assumptions of attribution theory in India. Joumal of Social Psychology, vol. 126 (5): 607-616.

Parsons, T., y Bales, R. F. (1955). Family, socialization and interaction process, Nueva York, Free Press.

Paulhus, D., y Christie, R. (1981). Spheres of Control: An interactionist approach to assessment of perceived control. H. M. Lefcourt (Ed.). Research with the Locus of Control Construct, Nueva York: Academic Press.

Scheier, M. F., y CARver, C. S. (1985). Self-consciousness scale: A revised version for use with general populations. Joumal of Applied Social Psychology, 15 (8), 687-699.

Tedesch.J. T. (1986). Private and public experiences and self. R. F. Baumeister (Ed.). Public self and private self, Nueva York; Springer-Verlag.

Tнотт, P. A. (1989). The sociology of emotions. Annual Review of Sociology, vol. 15: 317-342.

WEINER, B. (1986). Attribution, emotion and action. R. M. Sorrentino and E. T. Higgins (Eds.). Handbook of motivation and cognition, Nueva York: John Wiley \& Sons.

Weber, M. (1980). Economía y Sociedad, México, Fondo de Cultura Económica. 